\title{
Aldolase A Promotes Proliferation and Metastasis of Colorectal Cancer through Targeting COPS6 and Regulating MAPK Signaling Pathway
}

Ya Lu

affiliated Cancer Hospital of Nanjing Medical University https://orcid.org/0000-0002-3872-4683

Yuan Zhang

Jiangsu Cancer Hospital

Hui Zhang

Jiangsu Cancer Hospital

Yue Zhu

Nanjing Jinling Hospital

Junying Zhang

Jiangsu Cancer Hospital

Huanhuan Sha

Affiliated Cancer Hospital of Nanjing Medical University

Renrui Zou

Affiliated Cancer Hospital of Nanjing Medical University

Yujie Gan

Affiliated Cancer Hospital of Nanjing Medical University

Jianzhong Wu

Jiangsu Cancer Hospital

Jifeng Feng ( $\square$ fjif@jszlyy.com.cn )

Affiliated Cancer Hospital of Nanjing Medical University https://orcid.org/0000-0002-7979-2391

\section{Primary research}

Keywords: Colorectal cancer, Aldolase A, Proliferation, Invasion, Migration, COPS6, MAPK signaling pathway, EMT

Posted Date: August 24th, 2021

DOI: https://doi.org/10.21203/rs.3.rs-779219/v1

License: (c) (i) This work is licensed under a Creative Commons Attribution 4.0 International License. Read Full License 


\section{Abstract}

Background: Colorectal cancer (CRC) is a serious threat to human health, and its underlying mechanisms needs further explored. Aldolase A (ALDOA) has received increasing attention for its reported association with multiple cancers, but the function and mechanism of ALDOA in CRC remain unclear. We aimed to evaluate the biological role of ALDOA in CRC.

Methods: The stable ALDOA knockdown or overexpression cell lines were established for subsequent experiments. The qRT-PCR and western blotting were used to detect the expression of ALDOA and COPS6 and the relative protein levels of epithelial-mesenchymal transition (EMT) and MAPK signaling pathway. Immunofluorescence (IF) assay was applied to determine ALDOA localization. CCK-8, transwell, and wound healing assays were performed to evaluate CRC cell proliferation, invasion, and migration. Mouse xenograft models were established to verify the effect of ALDOA on CRC cell growth in vivo. Immunoprecipitation (IP) assay and mass spectrometry (MS) analysis were conducted to identify the interactions between ALDOA and COPS6.

Results: ALDOA was overexpressed in CRC tissues and cell lines. Silencing ALDOA significantly impaired the proliferation, invasion and migration of CRC cells in vitro, and obviously decreased the growth of CRC cells in vivo. Mechanically, ALDOA bound to and regulated COPS6, and the promoting effects of upregulated ALDOA on CRC cell proliferation and metastasis were inhibited by the depletion of COPS6. Besides, EMT program and MAPK signaling pathway were activated by ALDOA overexpression.

Conclusion: ALDOA facilitated the proliferation, invasion and migration of CRC through binding and regulating COPS6, inducing EMT and activating MAPK signaling pathway.

\section{Introduction}

Colorectal cancer (CRC) is one of the 4 leading cancers in the world[1], and has risen to the third place for malignant tumors in China[2]. Patients with advanced CRC usually have poor prognosis because of lacking of early diagnostic biomarkers and effective treatments $[3,4]$. Therefore, it is imperative to further explore the molecular mechanisms of CRC development and seek more reasonable and effective biological targets.

Increasing evidence suggested "cancer metabolism" is a new hallmark of cancers[5, 6]. Tumor cells tend to accelerate cancer progression through more efficient glycolysis rather than oxidative phosphorylation pathway[7]. Therefore, key enzymes involved in glycolysis are increasingly recognized as potential targets for cancer therapy[8].

Aldolase is the fourth enzyme in the glycolysis process, and its family members include aldolase A (ALDOA), aldolase $B(A L D O B)$ and aldolase $C$ (ALDOC), which are encoded by three different genes $[9,10]$. ALDOA is the major functional member among aldolase family due to its high activity and low $\mathrm{K}_{\mathrm{m}}$ value[9]. It is also an object of increased interest because of its high expression in various malignancies[11-13]. Research has revealed the importance of ALDOA in cancers, but the underlying mechanisms remain to be elucidated.

The aim of the present study was to evaluate the biological significance and function of ALDOA in CRC. It was discovered that ALDOA overexpression and high enzyme activity stimulate CRC cell growth and metastasis.

\section{Materials And Methods}

\section{Patient specimens collection and ethics statement}


CRC and adjacent normal tissue samples were collected from surgical specimens of CRC patients at the Affiliated Cancer Hospital of Nanjing Medical University (Nanjing, China). None of the patients had received chemotherapy, radiotherapy, and targeted therapy before tissue collection. The CRC diagnosis was confirmed by clinical criteria and pathological analysis. Informed consent was obtained from all subjects. All study protocols were approved by the Ethics Committee of Affiliated Cancer Hospital of Nanjing Medical University, China.

\section{Aldolase activity assay}

ALDOA activity of CRC specimens was measured using the Aldolase Activity Colorimetric Assay Kit (cat. no. K665100 , BioVision, Tucson, USA) according to the manufacturer's protocol. A total of $10 \mathrm{mg}$ of CRC tissue samples were rapidly homogenized in $100 \mu \mathrm{L}$ of ice-cold Aldolase Assay Buffer and kept on ice for $10 \mathrm{~min}$. After centrifuging at $10,000 \mathrm{~g}$ for $5 \mathrm{~min}, 5 \mu \mathrm{L}$ of sample supernatant and $50 \mu \mathrm{L}$ of reaction mix were added to a 96 -well plate. The absorbance $(450 \mathrm{~nm})$ was then immediately measured in kinetic mode for $45 \mathrm{~min}$ at $37^{\circ} \mathrm{C}$. One unit of Aldolase was the amount of enzyme that generated $1 \mu \mathrm{mol}$ of Nicotinamide adenine dinucleotide (NADH) per minute at pH 7.2 and $37^{\circ} \mathrm{C}$.

\section{Cell lines and culture conditions}

A total of five human CRC cell lines (DLD1, SW480, SW620, HCT116 and LoVo) and human normal colon epithelial cell line HCoEpiC used in this study were purchased from the American Type Culture Collection (ATCC). All cells lines were cultured in Dulbecco's Modified Eagle Medium (DMEM, KeyGEN BioTECH, Jiangsu, China) containing 10\% fetal bovine serum (FBS, Gibco, Thermo Fisher Scientific, Inc. Waltham, USA), $100 \mathrm{U} / \mathrm{mL}$ penicillin, $100 \mu \mathrm{g} / \mathrm{mL}$ streptomycin (Invitrogen, Thermo Fisher Scientific, Inc. Waltham, USA), and maintained in $37^{\circ} \mathrm{C}$ incubators with $5 \%$ $\mathrm{CO}_{2}$.

\section{RNA extraction and qRT-PCR analysis}

Total RNA samples were extracted from tissues or cultured cells using TRIZOL reagent (Invitrogen, Thermo Fisher Scientific, Inc.). The cDNA samples were synthesized using the Reverse Transcription Kit (TaKaRa, Tokyo, Japan) according to the manufacturer's guidelines. Quantitative real-time PCR (qRT-PCR) was used to detect the related genes using PowerUp SYBR Green Mix (Invitrogen, Thermo Fisher Scientific, Inc.). The 20- $\mu$ L reaction system consisted of $10 \mu \mathrm{L}$ of SYBR Green Mix, $1 \mu \mathrm{L}$ each of forward and reverse primers, $2 \mu \mathrm{L}$ of cDNA, and $6 \mu \mathrm{L}$ of double distilled water. Relative RNA expression was normalized to that of GAPDH. All qRT-PCR assays were performed in triplicate on ABI 7500 Fast Instrument (model 7300, Applied Biosystems, Thermo Fisher Scientific, Inc.). The primers were synthesized by Sangon Biotech (Shanghai, China). The primer sequences were as follows: ALDOA-F 5' GGTGCTGGCTGCTGTCTACAAG 3', ALDOA-R 5' GACGCCTCCTCCTCACTCTGG 3', ALDOB-F 5' AAGGCCCTGAATGACCATCA 3', ALDOB-R 5' GCATTGAGGTTGAGAGTGGC 3', ALDOC-F 5' AACCTCAATGCCATCAACCG 3', ALDOC-R 5' GCTCCACCATCTTCTCCACT 3', COPS6-F 5' ACCCTATGACCAAGCACACA 3', COPS6-R 5' TGCTATCAGGTGTTCAGCCA 3', GAPDH-F 5' GGATTTGGTCGTATTGGGCG 3', and GAPDH-R 5' ATCGCCCCACTTGATTTTGG 3'.

\section{Western blotting analysis and antibodies}

Protein samples were extracted from cells or tissues in RIPA lysis buffer (Invitrogen, Thermo Fisher Scientific, Inc.) containing a protease and phosphatase inhibitor cocktail (New Cell \& Molecular Biotech Co., Ltd, Suzhou, China). The amount of protein was quantified using the BCA Protein Assay Kit (Beyotime Biotechnology, Shanghai, China) according to the manufacturer's instructions. The protein extracts were then separated using $4 \%-12 \%$ gradient 
sodium dodecyl sulfate polyacrylamide gel electrophoresis (SDS-PAGE), transferred onto polyvinylidene difluoride membranes (Millipore, Bedford, MA), which were blocked with QuickBlock Blocking Buffer (Beyotime Biotechnology), and then incubated with primary and secondary antibodies. Protein bands were visualized using an ECL chemiluminescence reagent (New Cell \& Molecular Biotech Co., Ltd) and an Odyssey imaging system (LI-COR Biosciences). Protein levels were normalized using Tubulin (rabbit polyclonal, cat. no. 11224-1-AP, 1:2000 dilution, ProteinTech Group, Wuhan, China). The following antibodies were used: ALDOA (mouse monoclonal, cat. no. sc390733, 1:1000 dilution, Santa Cruz, California, USA), HRP-Conjugated DYKDDDDK Tag (monoclonal, cat. no. HRP66008, 1:5000 dilution, ProteinTech Group), E-cadherin (rabbit monoclonal, cat. no. 3195, 1:1000 dilution, Cell Signaling Technology, Boston,USA), N-cadherin (rabbit monoclonal, cat. no. 13116, 1:1000 dilution, Cell Signaling Technology), vimentin (rabbit monoclonal, cat. no. 5741, 1:1000 dilution, Cell Signaling Technology), p38 (rabbit monoclonal, cat. no. 8690, 1:1000 dilution, Cell Signaling Technology), p-p38 (rabbit monoclonal, cat. no. 8632, 1:1000 dilution, Cell Signaling Technology), ERK1/2 (rabbit monoclonal, cat. no. 4695, 1:1000 dilution, Cell Signaling Technology), p-ERK1/2 (rabbit monoclonal, cat. no. 4376, 1:1000 dilution, Cell Signaling Technology), ACTB (rabbit monoclonal, cat. no. AC038, 1:10000 dilution, ABclonal Technology, Wuhan, China), GAPDH (rabbit monoclonal, cat. no.60004-1-lg, 1:10000 dilution, ProteinTech Group), Lamin B1 (rabbit polyclonal, cat. no. 12987-1-AP, 1:2000 dilution, ProteinTech Group), PKM (rabbit polyclonal, cat. no. 10078-2-AP, 1:1000 dilution, ProteinTech Group), HSP90AB (rabbit polyclonal, cat. no. RK05737, 1:1000 dilution, ABclonal Technology), and CSN6 (mouse monoclonal, cat. no. sc-393023, 1:1000 dilution, Santa Cruz, California, USA).

\section{Immunofluorescence}

For immunofluorescence (IF) testing, the cells were fixed with 4\% paraformaldehyde for 10 min and then permeabilized with Triton X-100 for 15 min. ALDOA antibody (rabbit polyclonal, cat. no. A1142, 1:100 dilution, ABclonal Technology) was used for IF incubation, while 4,6-diamidino-2-phenylindole (Beyotime Biotechnology) was

added for cell nuclei staining. The fluorescent images were then captured under a fluorescence microscope (Olympus Corporation, Tokyo, Japan).

\section{Transfection of CRC cells}

Two individual ALDOA and one universal negative control small interfering RNA (siRNA) samples were purchased from RiboBio. The pcDNA3.1(+)-ALDOA overexpression plasmid was purchased from Public Protein/Plasmid Library (Jiangsu, China). The pcDNA3.1(+) empty vector was extracted using Endofree Plasmid Maxi Kit (Qiagen, Germany). The plasmid DNA or siRNAs were transiently transfected into CRC cells using Lipofectamine 3000 (Invitrogen, Thermo Fisher Scientific, Inc.) following the manufacturer's protocol.

\section{Construction of stable ALDOA knockdown or overexpression cell lines}

Lentiviral particles underexpressing or overexpressing ALDOA, both tagged with Green fluorescent protein (GFP), were purchased from Corues Biotechnology (Jiangsu, China). FLAG-tag was added to ALDOA-overexpressing lentiviral particles as well. These lentiviral particles were individually used to infect SW480 and DLD1 to generate the corresponding stable cell lines after purine screening for one week. The scrambled shRNA or empty vector transfected cells were established as matched controls. The efficiency of ALDOA knockdown or overexpression was assessed using western blot and qRT-PCR assays.

\section{Cell proliferation assay}


The cells were seeded in 96-well plates at a density of 3000 cells per well. Cell viability was evaluated using Cell Counting Kit-8 (CCK-8, Dojindo Laboratories, Tokyo, Japan) daily for three days. Then, 10\% CCK-8 solution was added to each well, and the cells were incubated for $1 \mathrm{~h}$. The absorbance was measured at a wavelength of $450 \mathrm{~nm}$ on SpectraMax (Molecular Devices).

\section{Wound healing assay}

The cells were cultured in six-well plates at a density of $5 \times 10^{5}$ cells $/ \mathrm{mL}$. After becoming confluent, the cells in the six-well plates were carefully scratched using $10-\mu \mathrm{L}$ sterile tips to form cell-free linear regions. The supernatant was then discarded after washing with phosphate buffer saline (KeyGEN BioTECH). The images were acquired using an inverted light microscope. Cell mobility was quantified by calculating the area of wound closure.

\section{Migration and invasion transwell assay}

Cell invasion assays were performed using Transwell chambers (Corning, New York, USA) precoated with Matrigel (BD Biocoat, Corning), while cell migration assays were completed without Matrigel. A total of 40,000 cells in $200 \mu \mathrm{L}$ of serum-free medium were seeded in the top chambers, while the lower chambers contained DMEM with $20 \%$ FBS. After $48 \mathrm{~h}$ of incubation at $37^{\circ} \mathrm{C}$, the cells migrated or invaded into the lower surface were fixed with $4 \%$ paraformaldehyde (Beyotime Biotechnology) and visualized using crystal violet staining (Beyotime Biotechnology). The images of cells were captured at $20 \times$ using a light microscope and three random fields were counted in each chamber. Three independent experiments were performed.

\section{Immunoprecipitation assay and mass spectrometry analysis}

Immunoprecipitation (IP) assays were performed using the Dynabead Protein G IP Kit (Thermo Fisher Scientific, Inc.) following the manufacturer's instructions. Anti-FLAG beads (Thermo Fisher Scientific, Inc.), stable ALDOA overexpression cell lines tagged with FLAG and their control cells, anti-ALDOA antibody (mouse monoclonal, cat. no. sc-390733, Santa Cruz, California, USA) and anti-COPS6 antibody (mouse monoclonal, cat. no. sc-393023, Santa Cruz, California, USA) were used in the IP assay. After the IP assay, 10\% SDS-PAGE gel electrophoresis was performed and a silver stain was applied using the Fast Silver Stain Kit (Beyotime Biotechnology) following the manufacturer's instructions. Proteins contained in the specific silver staining bands were identified using mass spectrometry (MS).

\section{Mouse xenograft assay}

Female BALB/c nude mice (4-6 weeks old) were purchased from Charles River Laboratory for CRC cell xenograft assay. A total of 12 nude mice were randomly assigned into two groups (ALDOA knockdown and control groups). A total of $6 \times 10^{6}$ cells $(150 \mu \mathrm{L})$ from each group were subcutaneously injected into the side of each nude mouse. The tumor volumes and body weight of nude mice were observed and monitored every three days. The tumor volume was calculated using the following formula: volume $\left(\mathrm{cm}^{3}\right)=\left(\right.$ length $\times$ width $\left.{ }^{2}\right) / 2$. After 30 days, the nude mice were placed into a container (clean and no $\mathrm{CO}_{2}$ prefilling) for euthanasia. Then, $100 \% \mathrm{CO}_{2}$ was introduced into the container using compressed $\mathrm{CO}_{2}$ gas cylinder with a $\mathrm{CO}_{2}$ flow controller. The flow rate was $10-30 \%$ of the container volume per minute (1.25-3.75 L/min). The $\mathrm{CO}_{2}$ exposure time was 2-3 min. After confirming that the nude mice were unconscious, on breathing, no heartbeat, and dilated pupils, the $\mathrm{CO}_{2}$ valve was closed, followed by observation for 2 min to ensure that the nude mice were dead. The tumors were immediately collected and measured, followed by hematoxylin-eosin (HE) staining and ki-67 detection (rabbit polyclonal, cat. no. 27309-1-AP, 1:8000 dilution, 
Proteintech Group). All animal experiments were approved by the Institutional Animal Care and Use Committee of Nanjing Medical University, Nanjing, China (No. 2006034).

\section{Statiatical analysis}

Data that were independently collected in triplicate are presented as means \pm standard deviation. All data were evaluated using one-way analysis of variance and Student's t-test with GraphPad Prism 7.0 software (GraphPad Software, Inc.). A $P$-value of $<0.05$ was considered statistically significant.

\section{Results}

\section{ALDOA is upregulated in CRC tissues and cell lines}

The qRT-PCR results showed that ALDOA was the main form of aldolase subtype in CRC cell lines (DLD1, SW480, SW620, HCT116 and LoVo) compared to ALDOB and ALDOC (Fig. 1A). The mRNA and protein expression of ALDOA was then assessed in $\mathrm{CRC}$ tissues $(\mathrm{N}=24)$. High ALDOA expression was detected both at mRNA and protein levels (Fig. 1B-D). Moreover,ALDOA enzyme activity in CRC tissues was significantly higher than that in the corresponding normal tissues (Fig. 1E). Compared to normal colon epithelial cells (HcoEpiC), ALDOA was overexoressed in CRC cell lines (Fig. 1F). In addition, IF and western blotting results showed that ALDOA was mainly expressed in the cytoplasm (Fig. 1G, H). These results revealed that ALDOA might be associated with CRC tumorigenesis.

\section{ALDOA knockdown inhibits CRC cell proliferation, invasion and migration in vitro}

In previous studies, little was known about the potential biological functions of ALDOA in CRC. For the stability of subsequent experiments, the stable ALDOA knockdown CRC cell lines were constructed in SW480 and DLD1. The efficiency of ALDOA was assessed both at mRNA and protein levels (Fig. 2A, B). Next, the results of CCK-8 (Fig. 2C), wound healing (Fig. 2D), and invasion and migration transwell (Fig. 2E) assays showed that CRC cell proliferation, invasion and migration abilities were significantly weakened after ALDOA was knocked down. At the same time, the inhibitory effect of ALDOA on the proliferation and metastasis of SW480 and DLD1 was also detected after being transfected with ALDOA siRNA (Fig. S1). These data suggested ALDOA served as an oncogene in the tumorigenesis and metastasis of CRC cells.

\section{ALDOA knockdown reduces CRC tumor growth in vivo}

To determine whether ALDOA expression can affect CRC tumors in vivo, DLD1 cells with stable ALDOA knockdown or control cells were subcutaneously injected into nude mice to establish the xenograft models. Consistent with the in vitro results, the stable ALDOA knockdown group showed remarkable inhibition of subcutaneous tumors compared to the control group (Fig. 3A-E). However, there was no statistically significant difference in body weight of nude mice between the two groups (Fig. 3F). In addition, HE and ki-67 staining showed lower tumor growth activity in the ALDOA knockdown group (Fig. 3G). Taken together, these results further indicated that ALDOA promoted CRC cell proliferation in vivo.

\section{ALDOA overexpression boots CRC cell proliferation, invasion and migration in vitro}

In order to further explore the biological behavior of ALDOA in the progression of CRC, ALDOA-overexpressing CRC cell lines were constructed using the matched plasmid/lentivirus. Ttheir efficiency was verified using qRT-PCR and western blotting assays (Fig. 4A, B, S2A, B). Eventually, consistent results were obtained in both transient and stable ALDOA overexpression cell lines. Upregulated ALDOA significantly enhanced the proliferation, invasion and migration 
activity of CRC cells (Fig. 4C-E, S2C-E). Therefore, the above results demonstrated that ALDOA performed an important function in facilitating CRC development.

\section{ALDOA binds to and interacts with COPS6}

Protein-protein interaction networks are important molecular mechanisms for protein-coding genes exerting biological roles. The IP assays, followed by MS analysis, were performed to identify the proteins that interact with ALDOA. In both SW480 and DLD1 cells, two protein bands interacted with flag-tagged ALDOA (Fig. 5A). MS analysis identified a total of 15 proteins and peptide fragments as the potential binding proteins for ALDOA (Fig. 5B). After endogenous (anti-ALDOA/COPS6) and exogenous (anti-Flag) ALDOA Co-IP-western blotting assays, ubiquitin-related protein COPS6, a subunit of COP9 signalosome, were confirmed as a binding partner of ALDOA (Fig. 5C-E).

Furthermore, qRT-PCR and western blotting results showed that mRNA and protein levels of COPS6 were reduced by ALDOA knockdown (Fig. 6A), while the expression of ALDOA were not been affected when COPS6 was knocked down (Fig. 6B). These data suggested the possibility that ALDOA could influence the occurrence and development of CRC through interacting with COPS6.

In addition, further MS analysis of ALDOA modification revealed multiple ubiquitination modification sites (Table 1), indicating that the deubiquitination enzyme (DUB) COPS6 might stabilize the expression of ALDOA via the interactions between them.

\section{COPS6 is a mediator of ALDOA's roles in CRC}

Based on the findings above, the biological function of COPS6 in CRC was next clarified. Firstly, COPS6 was highly expressed in CRC tissues, but there was no statistically significant difference in current small number of samples (Fig. 7A). Then, the inhibition of proliferation, invasion and migration of SW480 and DLD1 were detected after knocking down COPS6 with siRNA (Fig. 7B-D). We proposed a hypothesis that ALDOA could promote CRC proliferation and metastasis through upregulating COPS6.

To explore whether COPS6 was the downstream mediator through which ALDOA could affect CRC progression, rescue assays were performed. Results showed that COPS6 knockdown suppressed the promotion of proliferation, invasion and migration of CRC cells induced by overexpressed ALDOA (Fig. 8A-C). These data demonstrated that COPS6 served as a mediator of ALDOA to advance CRC progress.

\section{ALDOA promotes CRC progress through EMT and MAPK signaling pathway}

Epithelia-mesenchymal transition (EMT) is an important symbol of accelerating cancer progression and metastasis. It is also closely linked to the transformation of cytoskeletal proteins. The present study monitored the protein expression of EMT markers. Western blotting assays revealed that downregulated ALDOA or COPS6 markedly decreased the expression of N-cadherin and vimentin in both SW480 and DLD1, while the level of E-cadherin was increased (Fig. 9A, B), suggesting that ALDOA and COPS6 were the promoters of EMT in CRC.

Furthermore, the relevant signaling pathways were examined to elucidate the specific molecular mechanisms of ALDOA in CRC. Results showed that ALDOA knockdown significantly reduced the phosphorylation level of p38 and extracellular signal-regulated kinase 1/2 (ERK1/2) in CRC cells (Fig. 9C). Meanwhile, the activation of p38 and ERK1/2 signals inducing by overexpressed ALDOA was impaired by si-COPS6 (Fig. 9D). It fully indicated that the promoting role of ALDOA in CRC progression by mediating COPS6 was associated with mitogen-activated protein kinase (MAPK) signaling pathway. 


\section{Discussion}

The incidence and mortality of CRC have been increasing with changes in aging population, dietary habit, gut microbiota status and basic diseases[14,15]. According to the latest global cancer statistics, CRC is the third most commonly diagnosed cancer $(10.0 \%$ of the total cases) and the second leading cause of cancer deaths $(9.4 \%$ of the total cancer deaths) in both sexes combined. The pathogenesis and molecular mechanism of CRC have not yet been elucidated. Further exploration should be carried out to find more appropriate biomarkers and therapeutic targets for clinical diagnosis and treatment of CRC.

Accumulating studies have reported that cell metabolism reprogramming was inextricably related to tumorigenesis $[6,16]$. The increased lactic acid production and accelerated energy generation resulting from the glycolysis pathway created a favorable environment for cancer cell growth[7, 17]. ALDOA is a key enzyme in glycolysis that has been in the spotlight due to its association with the diagnosis, efficacy and prognosis of multiple cancers[18-22]. However, in CRC, the few previous studies on ALDOA were mostly limited to correlation analysis, little is known about its specific role and mechanism in CRC.The present study confirmed the higher expression and more enzyme activity of ALDOA in clinical specimens from CRC patients compared to corresponding normal tissues. Subsequently, biological function assays in vitro and in vivo were performed. ALDOA knockdown reduced CRC cell proliferation, invasion, and migration in vitro. Similarly, ALDOA silencing delayed the growth of subcutaneous CRC tumors in xenograft models. Consistent with these results, CRC cell proliferation, invasion, and migration were enhanced with ALDOA upregulation. These outcomes provide additional evidence to support the role of ALDOA in facilitating CRC progression.

Furthermore, the potential mechanisms of ALDOA in CRC progression were explored as well. Although ALDOA is a glycolytic enzyme, it has been reported to function in a non-enzymatic manner in cancers. For example, Chang et al. have found that ALDOA expedited the acquisition of lung cancer stemness by suppressing miR-145 expression and activating the Oct4/DUSP4/TRAF4 axis in the absence of aldolase enzyme activity[23]. Additionally, aldolase isomers have been recognized as novel regulators of oncogenic Wnt signaling pathway due to their GSK-3 $\beta$ complex leading to Axin membrane translocation[24]. Notably, ALDOA established a cancer-associated protein-protein interaction with $y$-actin, thereby influencing lung cancer cell metastasis[20]. Thus, IP assays and MS analysis were performed to better understand the molecular mechanisms behind ALDOA. The present study confirmed that ALDOA could interact with and regulate COPS6.

COPS6, namely COP9 signalosome subunit 6 (CSN6), is a subunit of the COP9 signalosome[25]. Previous studies have shown that COPS6 was abnormal overexpressed in many cancers and usually predicted tumor poor survival[2628]. This specificity of COPS6 has also been reported in CRC[29, 30]. In CRC, CSN6 was deregulated by EGFR signaling, in which ERK2 bound directly to CSN6 Leu163/Val165 and phosphorylated CSN6 at Ser148, and then CSN6 stabilized $\beta$-catenin level by blocking the ubiquitin-proteasome pathway, thereby promoting CRC development[30]. Herein, we directly indentified the role of COPS6 as a tumor promoter in CRC, and as a downstream mediator of ALDOA in controlling CRC progression. At the same time, we also observed that the expression of ALDOA was not influenced by COPS6. However, given that COPS6 is a DUB that has been widely reported to be involved in the ubiquitin-proteasome pathway, we proposed a hypothesis that COPS6 may stabilize ALDOA expression to some extent, thereby forming a positive feedback loop between them and further enhancing the impact on CRC progression. MS analysis on ALDOA modification sites revealed the presence of ubiquitination modification sites, which provided a bit of evidence for our hypothesis. Moreover, in addition to ubiquitination modification sites, phosphorylation, acetylation, methylation and other modification sites were also been found on ALDOA, indicating 
the existence of more diverse modification patterns. Further exploration and validation will help enrich the molecular mechanisms of ALDOA in CRC.

EMT has long been proposed to be a crucial mechanism during cancer progression and metastasis, capable of bestowing cancer cells with the ability to spread throughout the body[31, 32]. During this process, cancer cells lose their epithelial characteristics and gain mesenchymal properties[33, 34], showing cell adhesion weaken and cell motility enhanced[35]. EMT is characterized by the downregulation of epithelial cell junction proteins, for instance Ecadherin, and the activation of mesenchymal proteins, such as vimentin and $\mathrm{N}$-cadherin[35]. This present study detected the increased expression of E-cadherin and decreased level of $\mathrm{N}$-cadherin and vimentin in CRC cells with ALDOA and COPS 6 knockdown. It thus demonstrated that ALDOA and COPS6 could facilitate CRC metastasis through EMT programs.

MAPKs are serine-threonine protein kinases that involve in various cellular activities[36, 37]. MAPK pathway is one of the most essential signaling cascades of tumorogenesis including cancer cell proliferation, metastasis and other biological behaviors[38, 39]. MAPK signaling pathway includes p38 MAPK, c-Jun NH2-terminal kinase (JNK) and ERK in mammals [36]. Among them, the ERK MAPK pathway is the most important for cell proliferation and migration, and is usually located downstream of many growth-related genes[38]. As for p38 and JNK MAPK pathway, they are activated by multiple types of cellular stress and co-integrate signals that affect proliferation, differentiation, survival and migration[40]. We found that silenced ALDOA reduced the phosphorylation of p38 and ERK1/2, while the activation of $\mathrm{p} 38$ and ERK1/2 caused by ALDOA overexpression was partially rescued by knockdown COPS6 It illustrated that MAPK signaling pathway was an important part of ALDOA to affect CRC development through COPS6.

Taken together, these findings suggested that ALDOA overexpression promoted CRC cell proliferation, invasion and migration through interacting with COPS6, inducing EMT, and activating the MAPK signaling pathway. In the following work, the specific binding site between ALDOA and its target protein COPS 6 needs to be further confirmed, and whether COPS6 could influence the stability of ALDOA through some post-translational mechanism needs to be further explored. In addition, the further investigation is required for its clinical application as well.

\section{Conclusion}

In conclusion, our study certified that upregulated ALDOA boosted the progression of CRC by accelerating cell proliferation, EMT and metastasis via targeting COPS6 and regulating MAPK signaling pathway, providing a promising biomarker and therapeutic target for CRC.

\section{Abbreviations}




\begin{tabular}{|c|c|}
\hline Abbreviation & Full name \\
\hline CRC & colorectal cancer \\
\hline ALDOA & Aldolase A \\
\hline ALDOB & Aldolase B \\
\hline ALDOC & Aldolase C \\
\hline ATCC & American Type Culture Collection \\
\hline DMEM & Dulbecco's Modified Eagle Medium \\
\hline FBS & fetal bovine serum \\
\hline qRT-PCR & quantitative real-time PCR \\
\hline SDS-PAGE & sodium dodecyl sulfate polyacrylamide gel \\
\hline PVDF & polyvinylidene difluoride \\
\hline GAPDH & glyceraldehyde 3-phosphate dehydrogenase \\
\hline EMT & Epithelial-mesenchymal transition \\
\hline ERK1/2 & Extracellular Signal-egulated Kinase1/2 \\
\hline MAPK & mitogen-activated protein kinase \\
\hline PKM & pyruvate kinase $\mathrm{M} 1 / 2$ \\
\hline HSP90AB & heat shock protein 90 \\
\hline COPS6/CSN6 & COP9 signalosome subunit 6 \\
\hline IF & immunofluorescence \\
\hline GFP & green fluorescent protein \\
\hline CCK-8 & cell counting kit-8 \\
\hline PBS & phosphate buffer saline \\
\hline IP & immunoprecipitation \\
\hline MS & mass spectrometry \\
\hline HE & hematoxylin-eosin \\
\hline DUB & deubiquitination enzyme \\
\hline Oct4 & recombinant octamer binding transcription factor 4 \\
\hline DUSP4 & dual specificity phosphatase 4 \\
\hline TRAF4 & TNF receptor associated factor 4 \\
\hline
\end{tabular}

\section{Declarations}

Ethics approval and consent to participate 
The studies involving human participants were reviewed and approved by the Ethics Committee of Affiliated Cancer Hospital of Nanjing Medical University. The patients/participants provided their written informed consent to participate in this study. The animal study was reviewed and approved by the Institutional Animal Care and Use Committee (IACUC) of Nanjing Medical University (No. 2006034).

\section{Consent for publication}

Not applicable

\section{Availability of data and materials}

The datasets used and/or analysed during the current study are available from the corresponding author on reasonable request.

\section{Competing interests}

The authors have declared that no competing interest exists.

\section{Funding}

This study was supported by Youth Program of National Natural Science Foundation of China (No. 81902489), National Key R\&D Program of China (No. 2017YFC0114303) and Jiangsu Province's Key Discipline of Medicine (No. ZDXKA2016009).

\section{Author contributions}

JF and JW designed and funded the study. YL performed the assays and wrote the manuscript. All the authors were engaged into data analysis and literal editing. All authors read and approved the final manuscript.

\section{Acknowledgements}

Not applicable.

\section{References}

1. Siegel RL, Miller KD, Fuchs HE, Jemal A: Cancer Statistics, 2021. CA Cancer J Clin 2021, 71(1):7-33.

2. Cao M, Li H, Sun D, Chen W: Cancer burden of major cancers in China: A need for sustainable actions. Cancer Commun (Lond) 2020, 40(5):205-210.

3. Cronin KA, Lake AJ, Scott S, Sherman RL, Noone AM, Howlader N, Henley SJ, Anderson RN, Firth AU, Ma J et al: Annual Report to the Nation on the Status of Cancer, part I: National cancer statistics. Cancer 2018, 124(13):2785-2800.

4. Chen H, Li N, Ren J, Feng X, Lyu Z, Wei L, Li X, Guo L, Zheng Z, Zou S et al: Participation and yield of a population-based colorectal cancer screening programme in China. Gut 2019, 68(8):1450-1457.

5. Hanahan D, Weinberg RA: Hallmarks of cancer: the next generation. Cell 2011, 144(5):646-674.

6. Cantor JR, Sabatini DM: Cancer cell metabolism: one hallmark, many faces. Cancer Discov 2012, 2(10):881-898.

7. Liberti MV, Locasale JW: The Warburg Effect: How Does it Benefit Cancer Cells? Trends Biochem Sci 2016, 41(3):211-218. 
8. Ganapathy-Kanniappan S, Geschwind JF: Tumor glycolysis as a target for cancer therapy: progress and prospects. Mol Cancer 2013, 12:152.

9. Chang YC, Yang YC, Tien CP, Yang CJ, Hsiao M: Roles of Aldolase Family Genes in Human Cancers and Diseases. Trends Endocrinol Metab 2018, 29(8):549-559.

10. Kukita A, Yoshida MC, Fukushige S, Sakakibara M, Joh K, Mukai T, Hori K: Molecular gene mapping of human aldolase A (ALDOA) gene to chromosome 16. Hum Genet 1987, 76(1):20-26.

11. Chang YC, Chan YC, Chang WM, Lin YF, Yang CJ, Su CY, Huang MS, Wu ATH, Hsiao M: Feedback regulation of ALDOA activates the HIF-1alpha/MMP9 axis to promote lung cancer progression. Cancer Lett 2017, 403:28-36.

12. Ji S, Zhang B, Liu J, Qin Y, Liang C, Shi S, Jin K, Liang D, Xu W, Xu H et al: ALDOA functions as an oncogene in the highly metastatic pancreatic cancer. Cancer Lett 2016, 374(1):127-135.

13. Shimizu T, Inoue K, Hachiya H, Shibuya N, Shimoda M, Kubota K: Frequent alteration of the protein synthesis of enzymes for glucose metabolism in hepatocellular carcinomas. J Gastroenterol 2014, 49(9):1324-1332.

14. Dekker E, Tanis PJ, Vleugels JLA, Kasi PM, Wallace MB: Colorectal cancer. Lancet 2019, 394(10207):1467-1480.

15. Yang Y, Han Z, Li X, Huang A, Shi J, Gu J: Epidemiology and risk factors of colorectal cancer in China. Chin J Cancer Res 2020, 32(6):729-741.

16. DeBerardinis RJ, Lum JJ, Hatzivassiliou G, Thompson CB: The biology of cancer: metabolic reprogramming fuels cell growth and proliferation. Cell metabolism 2008, 7(1):11-20.

17. Estrella V, Chen T, Lloyd M, Wojtkowiak J, Cornnell HH, Ibrahim-Hashim A, Bailey K, Balagurunathan Y, Rothberg JM, Sloane BF et al: Acidity generated by the tumor microenvironment drives local invasion. Cancer Res 2013, 73(5):1524-1535.

18. Dai L, Pan G, Liu X, Huang J, Jiang Z, Zhu X, Gan X, Xu Q, Tan N: High expression of ALDOA and DDX5 are associated with poor prognosis in human colorectal cancer. Cancer Manag Res 2018, 10:1799-1806.

19. Li J, Wang F, Gao H, Huang S, Cai F, Sun J: ALDOLASE A regulates invasion of bladder cancer cells via Ecadherin-EGFR signaling. J Cell Biochem 2019, 120(8):13694-13705.

20. Chang YC, Chiou J, Yang YF, Su CY, Lin YF, Yang CN, Lu PJ, Huang MS, Yang CJ, Hsiao M: Therapeutic Targeting of Aldolase A Interactions Inhibits Lung Cancer Metastasis and Prolongs Survival. Cancer Res 2019, 79(18):4754-4766.

21. Kawai K, Uemura M, Munakata K, Takahashi H, Haraguchi N, Nishimura J, Hata T, Matsuda C, Ikenaga M, Murata $\mathrm{K}$ et al: Fructose-bisphosphate aldolase $\mathrm{A}$ is a key regulator of hypoxic adaptation in colorectal cancer cells and involved in treatment resistance and poor prognosis. Int J Oncol 2017, 50(2):525-534.

22. Jiang Z, Wang X, Li J, Yang H, Lin X: Aldolase A as a prognostic factor and mediator of progression via inducing epithelial-mesenchymal transition in gastric cancer. J Cell Mol Med 2018, 22(9):4377-4386.

23. Chang YC, Yang YF, Chiou J, Tsai HF, Fang CY, Yang CJ, Chen CL, Hsiao M: Nonenzymatic function of Aldolase A downregulates miR-145 to promote the Oct4/DUSP4/TRAF4 axis and the acquisition of lung cancer stemness. Cell Death Dis 2020, 11(3):195.

24. Caspi M, Perry G, Skalka N, Meisel S, Firsow A, Amit M, Rosin-Arbesfeld R: Aldolase positively regulates of the canonical Wnt signaling pathway. Mol Cancer 2014, 13:164.

25. Qin B, Zou S, Li K, Wang H, Wei W, Zhang B, Xiao L, Choi HH, Tang Q, Huang D et al: CSN6-TRIM21 axis instigates cancer stemness during tumorigenesis. Br J Cancer 2020, 122(11):1673-1685.

26. Forozan F, Mahlamaki EH, Monni O, Chen Y, Veldman R, Jiang Y, Gooden GC, Ethier SP, Kallioniemi A, Kallioniemi OP: Comparative genomic hybridization analysis of 38 breast cancer cell lines: a basis for interpreting 
complementary DNA microarray data. Cancer Res 2000, 60(16):4519-4525.

27. Chen J, Shin JH, Zhao R, Phan L, Wang H, Xue Y, Post SM, Ho Choi H, Chen JS, Wang E et al: CSN6 drives carcinogenesis by positively regulating Myc stability. Nature communications 2014, 5:5384.

28. Shi J, Guan X, Zhan F, Liu C, Li Z, Yao Y, Wang B, Lou C, Zhang Y: CSN6 expression is associated with pancreatic cancer progression and predicts poor prognosis. Cancer Biol Ther 2019, 20(9):1290-1299.

29. Yang L, Liu Y, Wang M, Qian Y, Dong X, Gu H, Wang H, Guo S, Hisamitsu T: Quercetin-induced apoptosis of HT-29 colon cancer cells via inhibition of the Akt-CSN6-Myc signaling axis. Mol Med Rep 2016, 14(5):4559-4566.

30. Fang L, Lu W, Choi HH, Yeung SC, Tung JY, Hsiao CD, Fuentes-Mattei E, Menter D, Chen C, Wang L et al: ERK2Dependent Phosphorylation of CSN6 Is Critical in Colorectal Cancer Development. Cancer Cell 2015, 28(2):183197.

31. Singh M, Yelle N, Venugopal C, Singh SK: EMT: Mechanisms and therapeutic implications. Pharmacol Ther 2018, 182:80-94.

32. Pradella D, Naro C, Sette C, Ghigna C: EMT and stemness: flexible processes tuned by alternative splicing in development and cancer progression. Mol Cancer 2017, 16(1):8.

33. Kang Y, Massague J: Epithelial-mesenchymal transitions: twist in development and metastasis. Cell 2004, 118(3):277-279.

34. Singh A, Settleman J: EMT, cancer stem cells and drug resistance: an emerging axis of evil in the war on cancer. Oncogene 2010, 29(34):4741-4751.

35. Vu T, Datta PK: Regulation of EMT in Colorectal Cancer: A Culprit in Metastasis. Cancers (Basel) 2017, 9(12).

36. Kim EK, Choi EJ: Compromised MAPK signaling in human diseases: an update. Arch Toxicol 2015, 89(6):867882.

37. Peti W, Page R: Molecular basis of MAP kinase regulation. Protein Sci 2013, 22(12):1698-1710.

38. Fang JY, Richardson BC: The MAPK signalling pathways and colorectal cancer. Lancet Oncol 2005, 6(5):322327.

39. Burotto M, Chiou VL, Lee JM, Kohn EC: The MAPK pathway across different malignancies: a new perspective. Cancer 2014, 120(22):3446-3456.

40. Wagner EF, Nebreda AR: Signal integration by JNK and p38 MAPK pathways in cancer development. Nat Rev Cancer 2009, 9(8):537-549.

\section{Tables}

Table 1: Post-translational modification sites in ALDOA 


\begin{tabular}{|llllll|}
\hline Modification & Sequence & MH+ & Charge & Score & ExpectValue \\
\hline GlyGly (K) & R.CQYVTEK\#VLAAVYK.A & 1785.92036 & 3 & 51.86 & 0.000000977 \\
& R.ALQASALK\#AWGGK.K & 1414.78009 & 2 & 39.76 & 0.0000159 \\
& K.VDK\#GVVPLAGTNGETTTQGLDGLSER.C & 2728.37481 & 3 & 39.08 & 0.0000309 \\
\hline $\begin{array}{l}\text { Phospho } \\
\text { (ST) }\end{array}$ & K.VDKGVVPLAGT^NGETTTQGLDGLSER.C & 1691.84212 & 3 & 48.22 & 0.00000301 \\
& K.GVVPLAGT^NGETTTQGLDGLSER.C & 2352.10789 & 3 & 41.91 & 0.000178 \\
\hline & R.ALANS^LACQGK.Y & 1212.54422 & 2 & 37.79 & 0.00000032 \\
\hline Acetyl (K) & K.DGADFAK*WR.C & 1107.52177 & 2 & 52.96 & 0.000000253 \\
\hline Methyl (DE) & R.IVAPGKGILAAD@E@STGSIAK.R & 1926.0906 & 2 & 20.49 & 0.0000893 \\
\hline & K.VD@KGVVPLAGTNGE@TTTQGLDGLSER.C & 2642.3632 & 3 & 20.13 & 0.00243 \\
\hline
\end{tabular}

\#: Ubiquitination site, ^: Phosphorylation site, *: Acetylation site, @: Methylation site

\section{Figures}


A

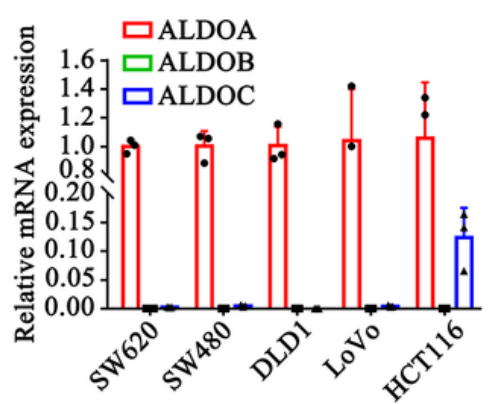

B

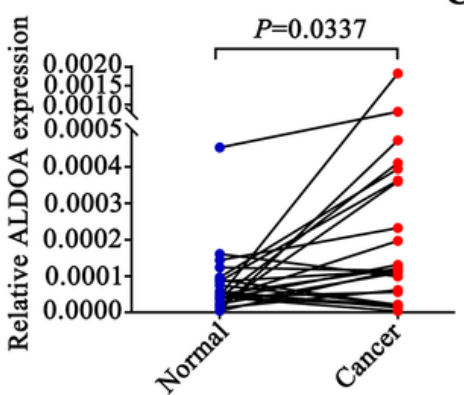

C

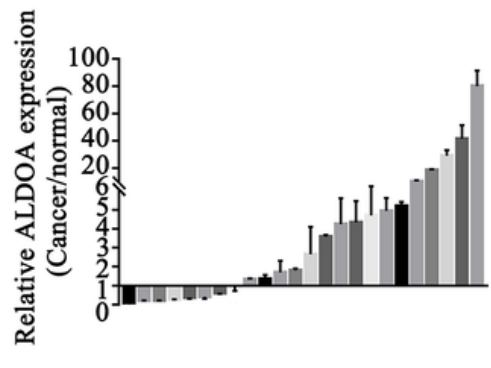

D

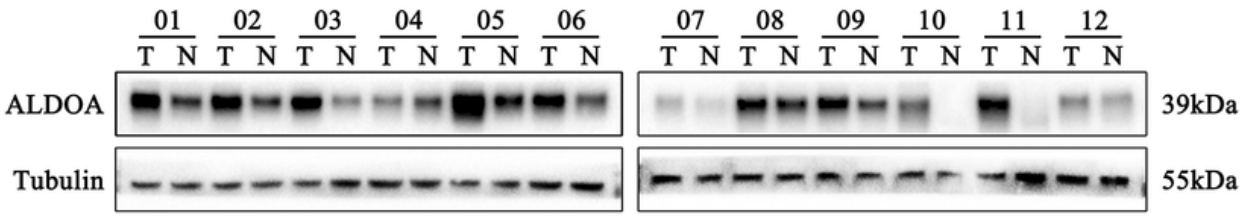

E

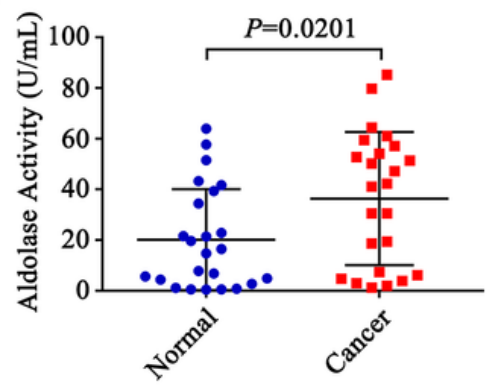

G
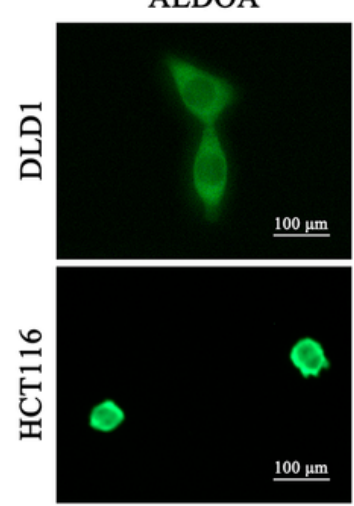

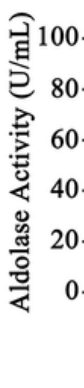

DAPI
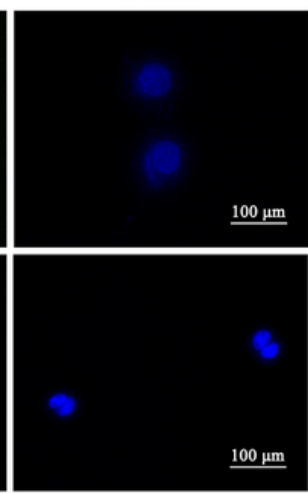

F :

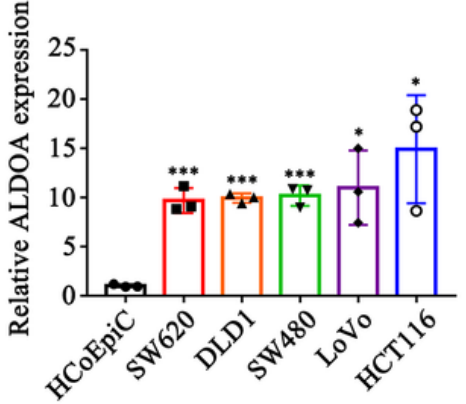

H
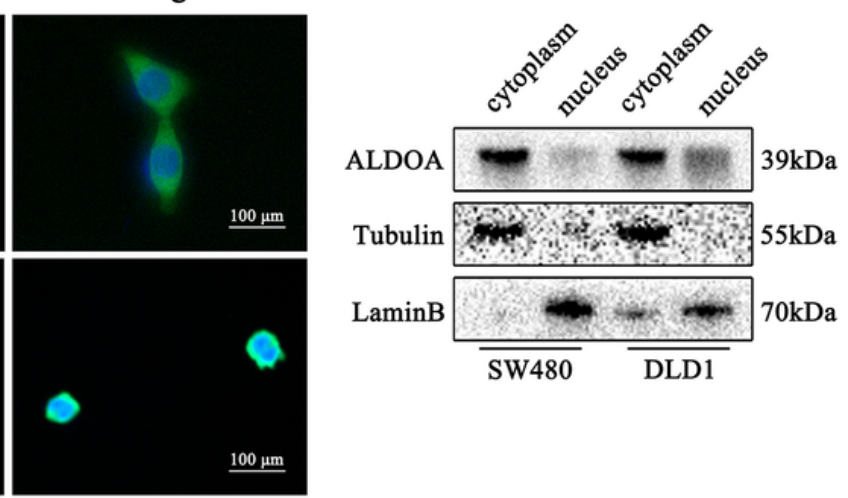

Figure 1

ALDOA was overexpressed in CRC tissues and cell lines. (A) ALDOA, ALDOB and ALDOC were examined by qRT-PCR in $C R C$ cell lines and ALDOA occupied the most expression in aldolase family. $(B, C)$ The overexpressed mRNA expression of ALDOA was tested in CRC tissues. (D) Western blotting showed elevated protein expression of ALDOA in CRC tissues. (E) The enzyme activity of ALDOA in CRC tissues was assessed higher level than normal. (F) Upregulation of ALDOA mRNA levels in CRC cell lines (SW620, DLD1, SW480, LoVo, HCT116) was examined by qRTPCR. (G) The localization of ALDOA was observed mainly in cytoplasm by IF assay. $(\mathrm{H})$ Western blotting detected

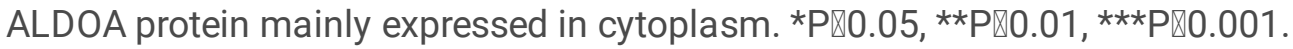


$\mathbf{A}$

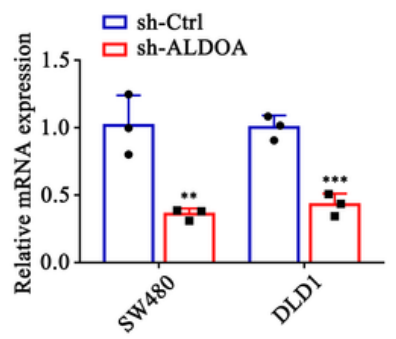

C

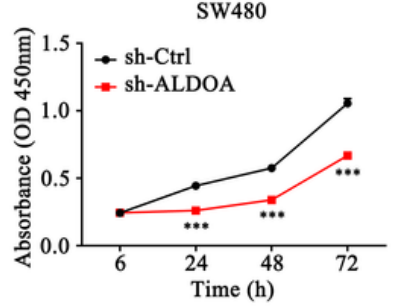

B

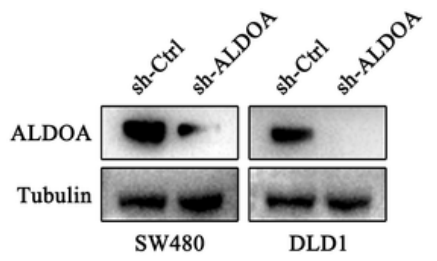

DLD1

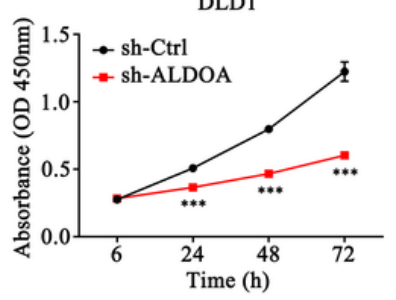

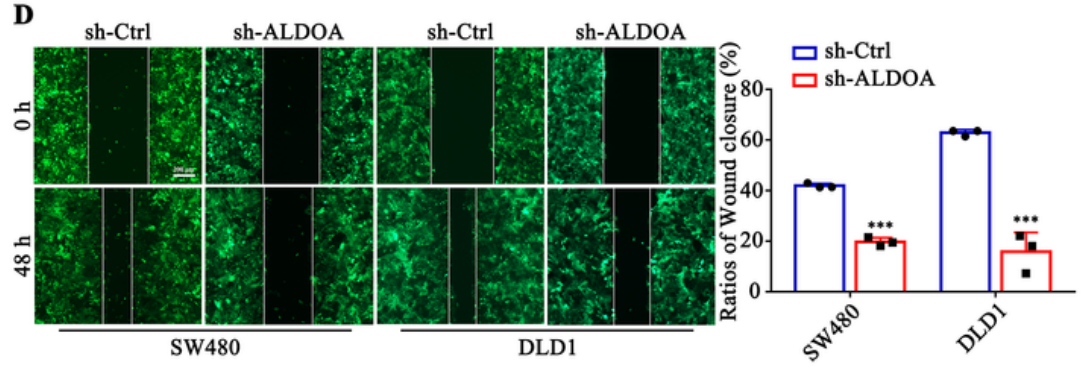

$\mathbf{E}$
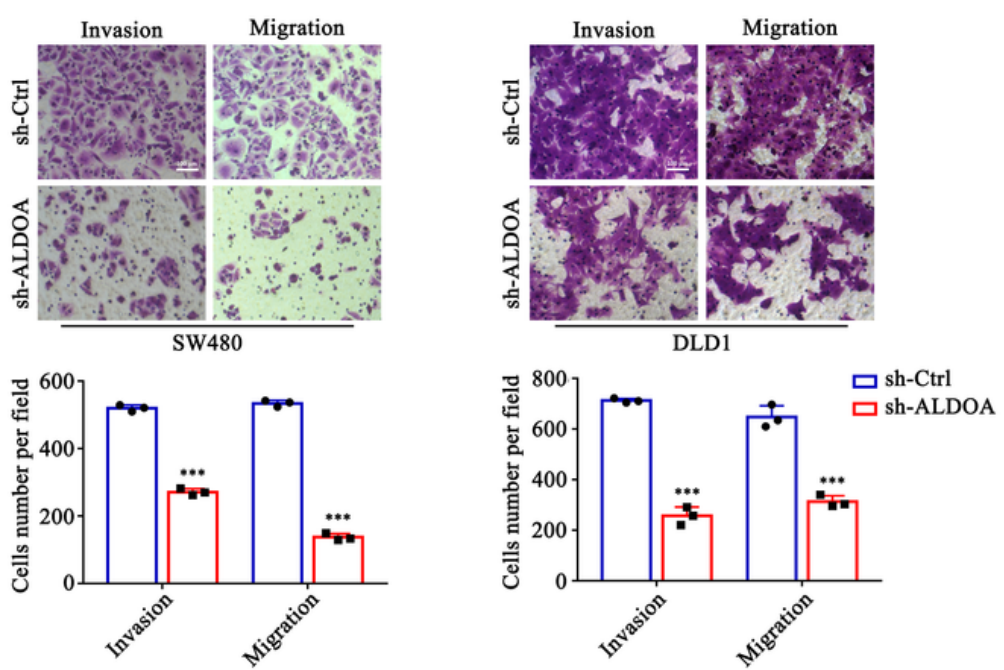

Figure 2

Knockdown ALDOA inhibited proliferation and metastasis of CRC cells in vitro. (A, B) The qRT-PCR and western blotting analysis were used to tested the knockdown efficiency of ALDOA. (C) Knockdown of ALDOA suppressed proliferation ability of SW480 and DLD1 cells using CCK-8 assays. (D) ALDOA shRNA inhibited CRC cell migration according to wound healing assays. (E) Transwell assays, with matrigel or not, were applied to assess the capability of $\mathrm{CRC}$ cells on invasion and migration. ${ }^{*} \mathrm{P} \otimes 0.05, * * P \llbracket 0.01, * \star \star P \llbracket 0.001$. 
$\mathbf{A}$
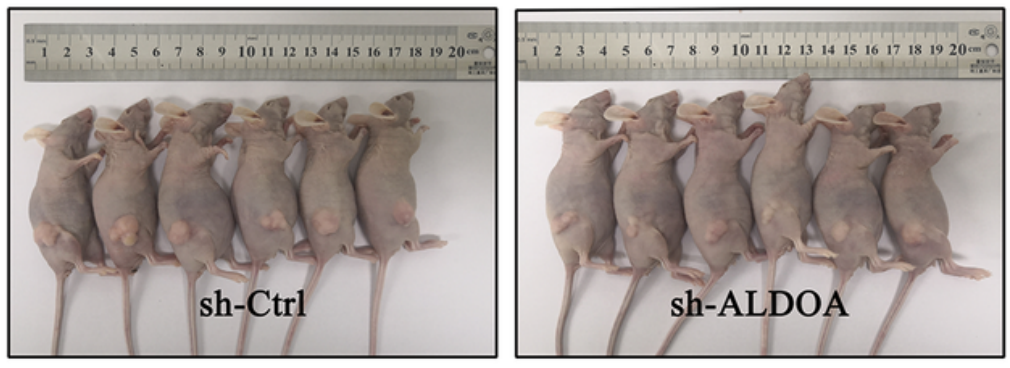

B

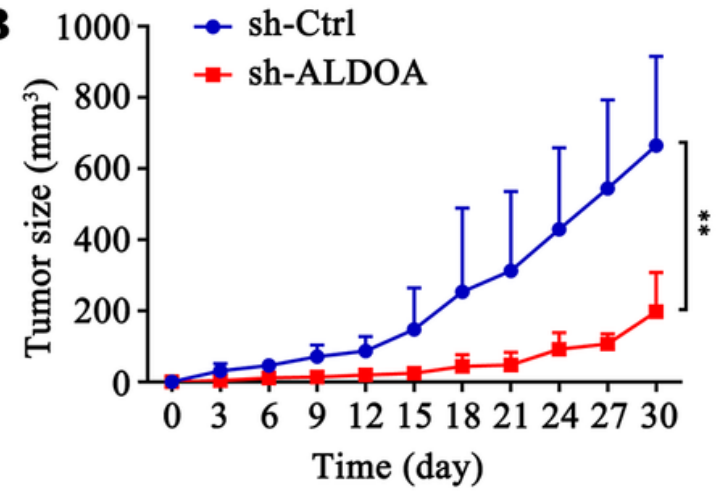

C

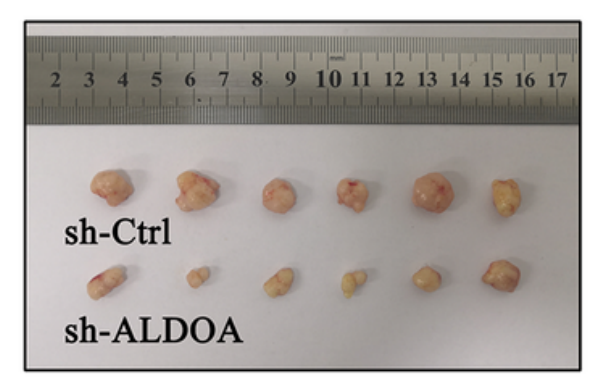

D

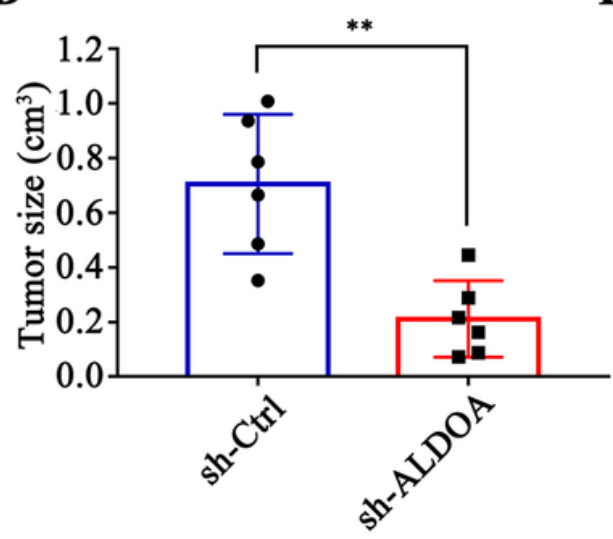

G

$\mathbf{F}$

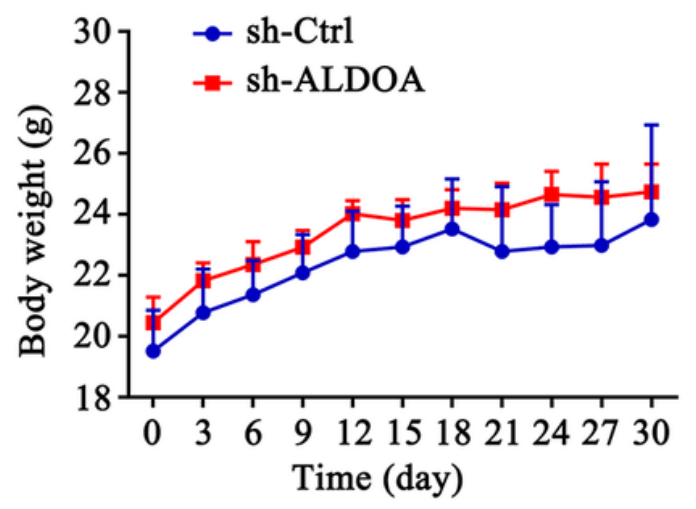

$\mathbf{E}$

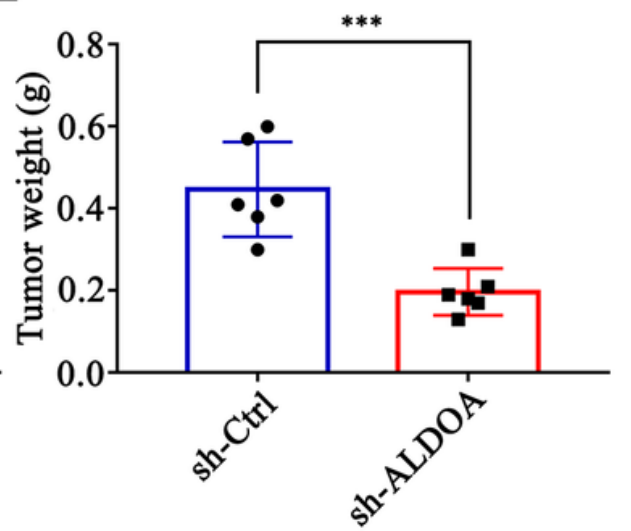

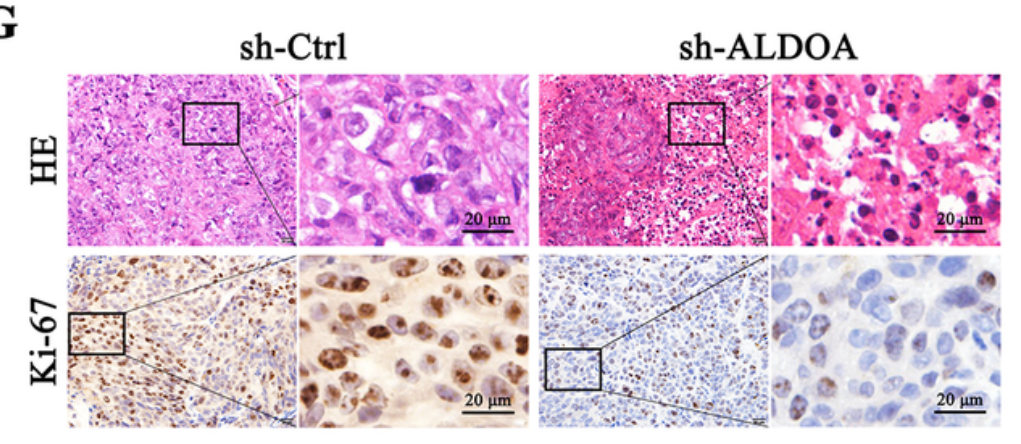

Figure 3

ALDOA facilitated CRC growth in vivo. (A) Morphologic characteristics of CRC xenograft tumors on the surface of nude mice. (B) The volume of subcutaneous tumors in mice was measured every three days.

Volume=length $\times$ width2/2. (C) An intuitive morphology of tumors collected after mice were sacrificed. (D, E) Tumor volumes and weights at 30 day were measured in each group. (F) The nude mice were weighed every three days, but no significant difference was observed. (G) Representative images of HE and ki-67 staining in CRC xenograft tumors from sh-Ctrl and sh-ALDOA nude mice. ${ }^{*} \mathrm{P} \otimes 0.05,{ }^{*} \mathrm{P} \otimes 0.01,{ }^{* \star * P} \llbracket 0.001$. 
$\mathbf{A}$

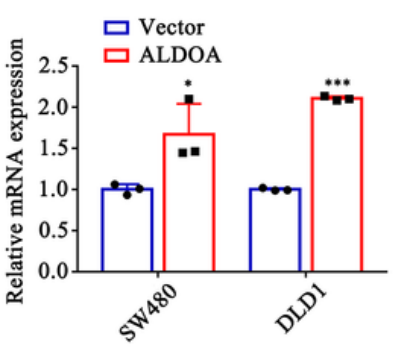

$\mathbf{C}$

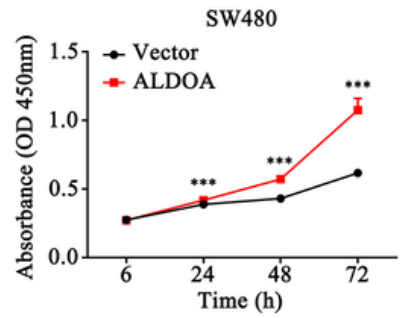

B

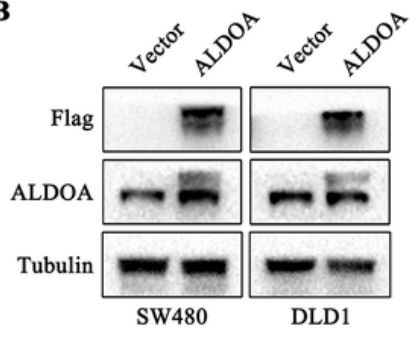

DLD1

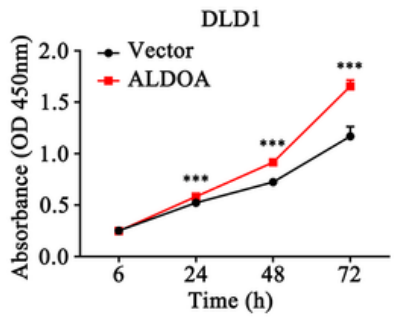

D

Vector

ALDOA

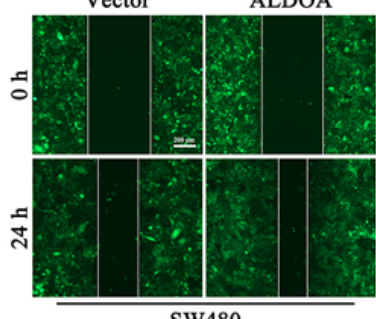

Vector
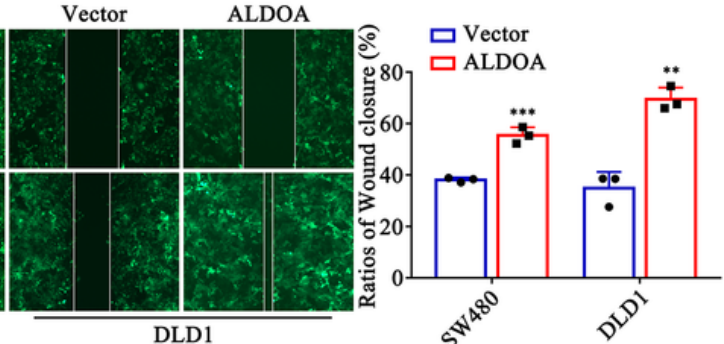

$\mathbf{E}$
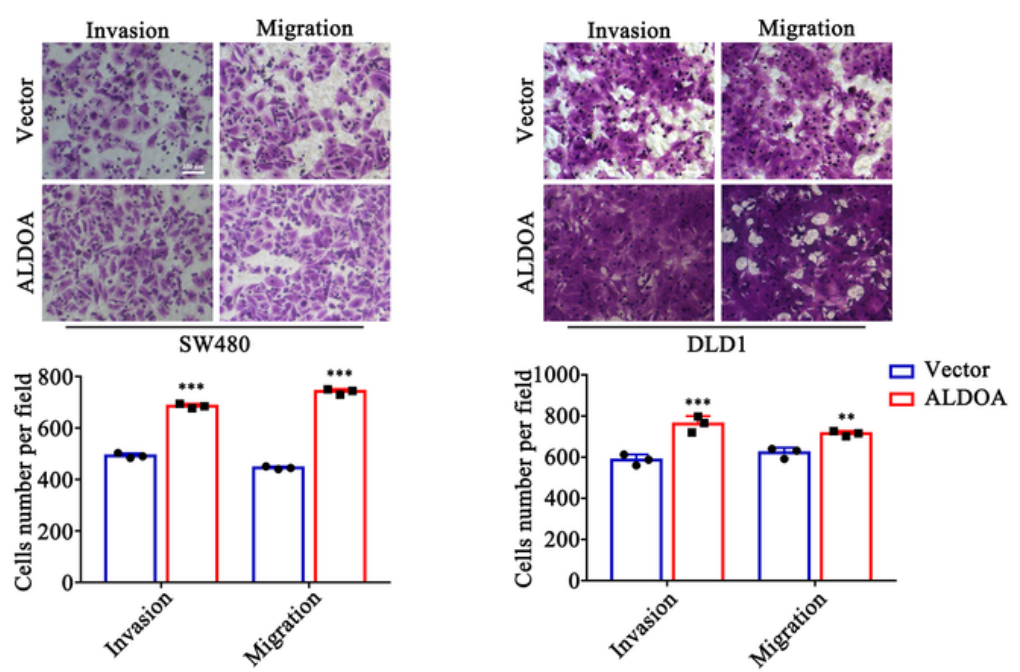

Figure 4

Overexpressed ALDOA enhanced the proliferation and metastasis of CRC cells. (A, B) The upregulated effect of stable ALDOA overexpression CRC cell lines was identified by qRT-PCR and western blotting analysis. (C) CCK-8 assays showed that upregulation of ALDOA promoted CRC cell proliferation. (D) Wound healing assays demonstrated increased ALDOA was beneficial to CRC cell migration. (E) Matrigel-transwell and non-matrigeltranswell assays respectively verified overexpressed ALDOA enhanced the invasion and migration of CRC cells. ${ }^{*} \mathrm{P} \triangle$ $0.05, * * \mathrm{P} \otimes 0.01, * \star * \mathrm{P} \otimes 0.001$. 
$\mathbf{A}$

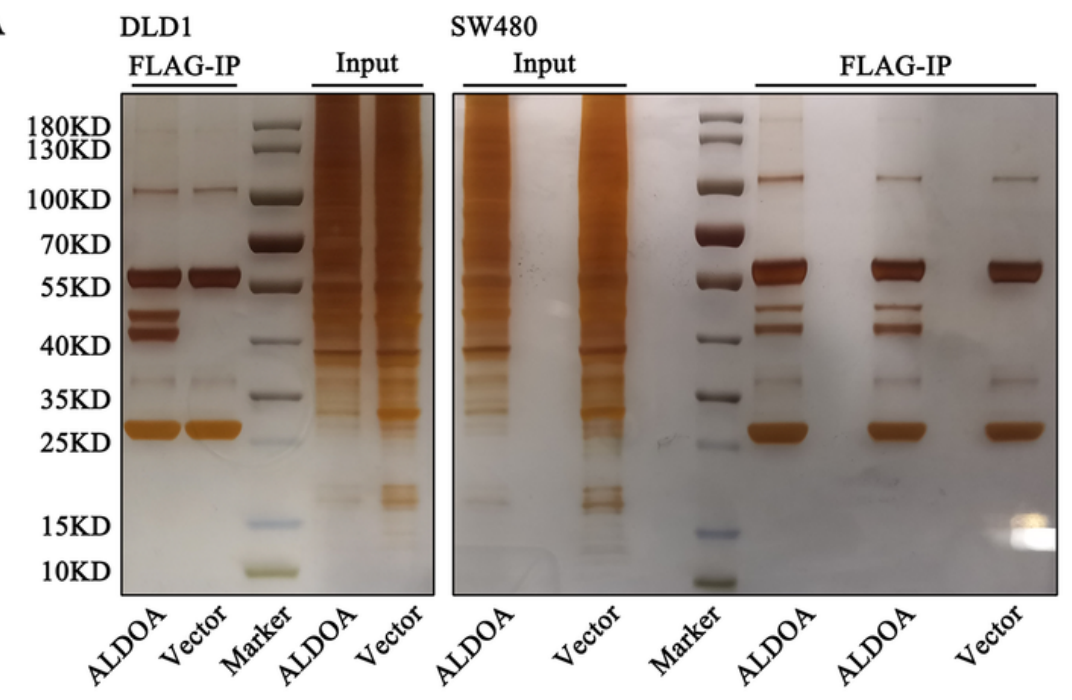

SW480

B

$\mathbf{C}$

IP:
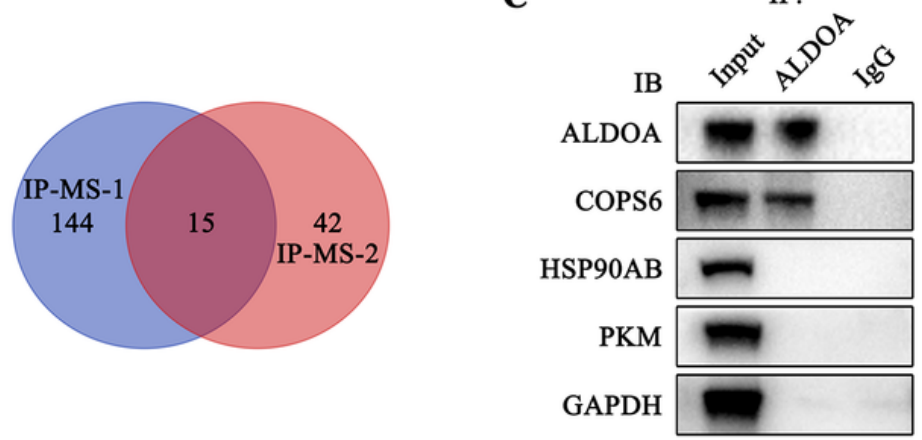

D

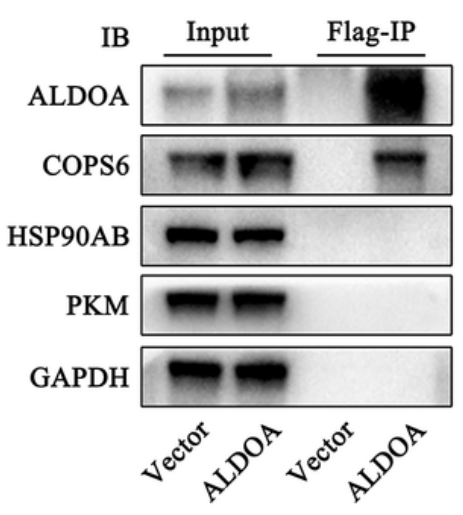

$\mathbf{E}$

IP:

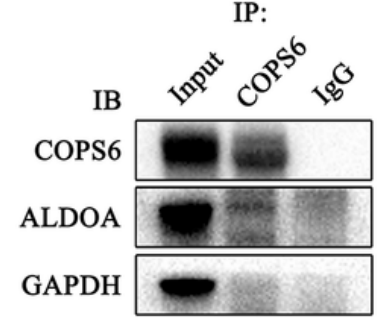

\section{Figure 5}

Protein-protein binding relationship between ALDOA and COPS6. (A) In stable overexpressed ALDOA cell lines tagged with flag, utilizing the characteristic of specific binding to flag beads, IP assays were performed to identify the interactive proteins that could bind to ALDOA. (B) Fifteen protein molecules and fragments were found to have the potential to bind to ALDOA according to the two MS analysis. (C-E) IP assays and western blotting were performed to confirmed the binding relationship between ALDOA and COPS6. 
$\mathbf{A}$

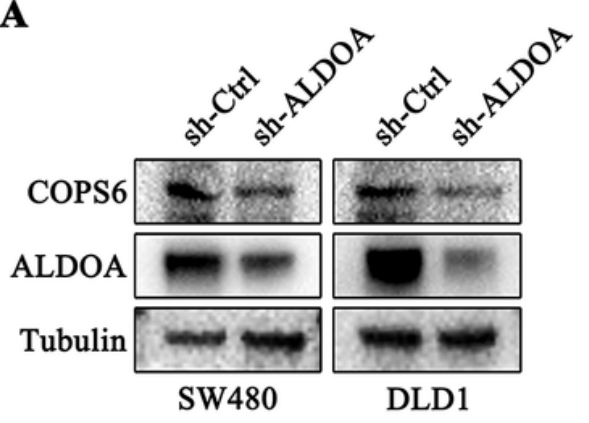

$\mathbf{C}$

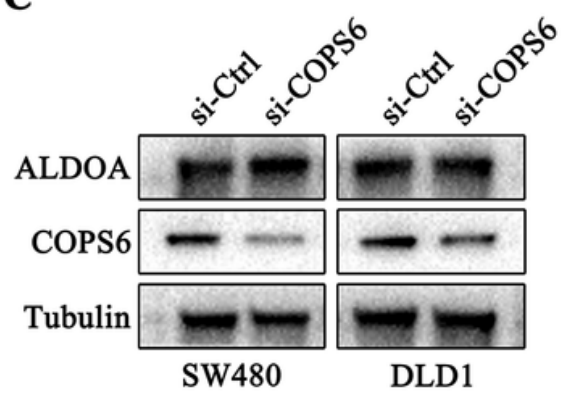

B

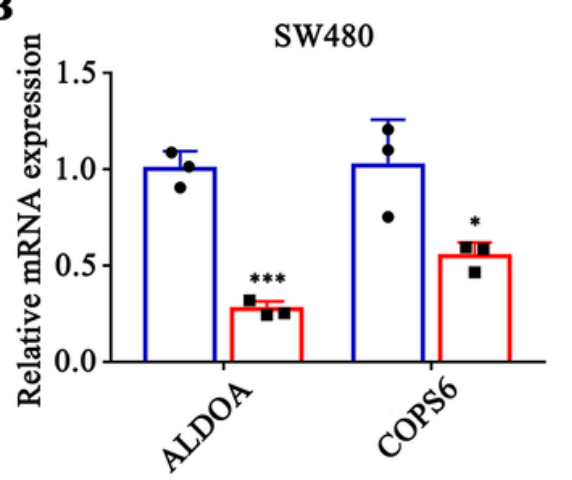

D

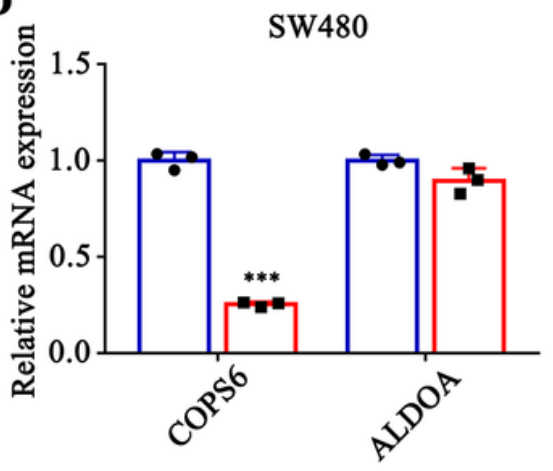

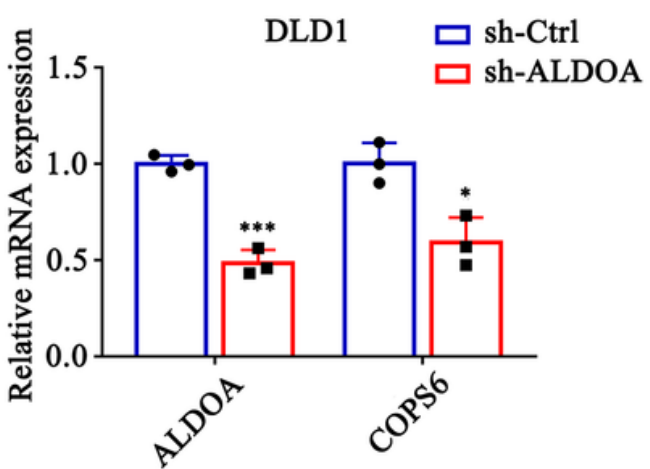

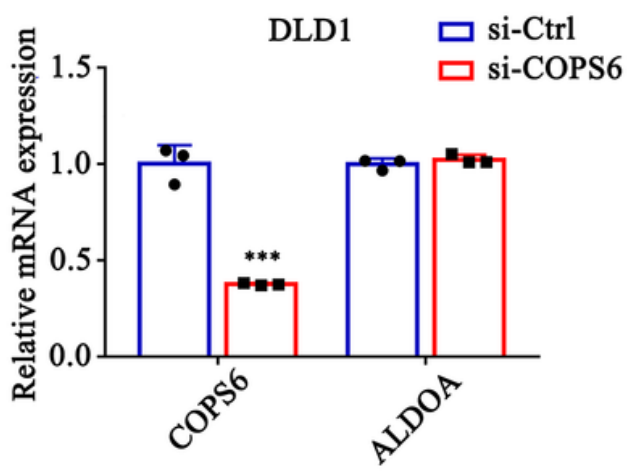

Figure 6

ALDOA regulated the expression of COPS6. $(A, B)$ Western blotting and qRT-PCR analysis were used to assess the protein and mRNA levels of COPS6 in stable knockdown ALDOA cell lines. (C, D) The expression of ALDOA was

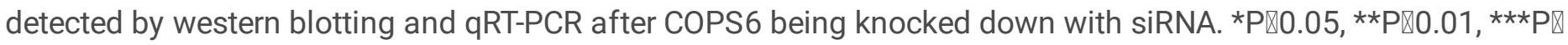
0.001 . 
$\mathbf{A}$
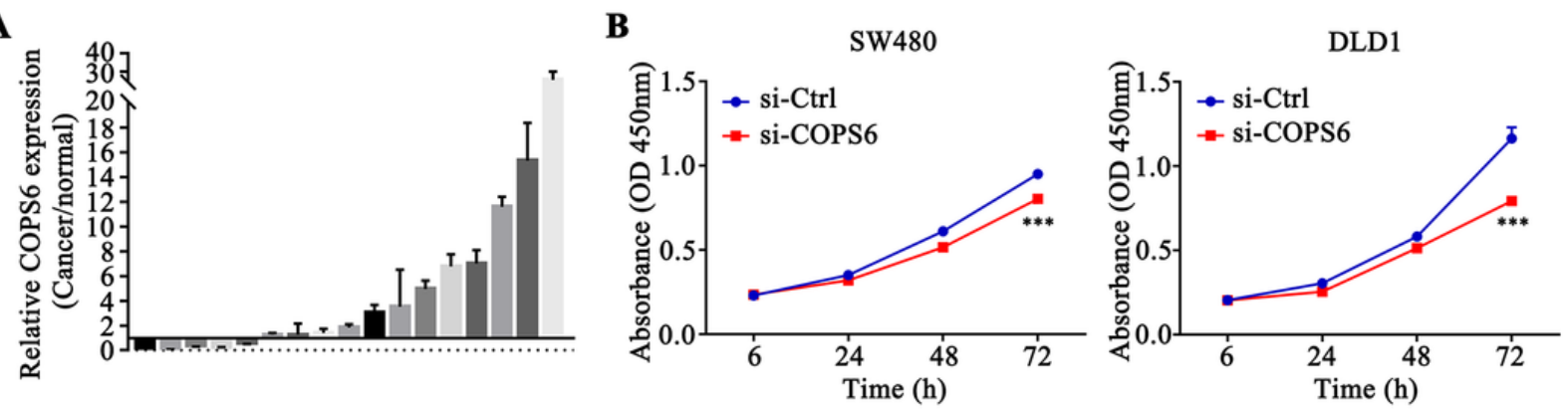

$\mathbf{C}$
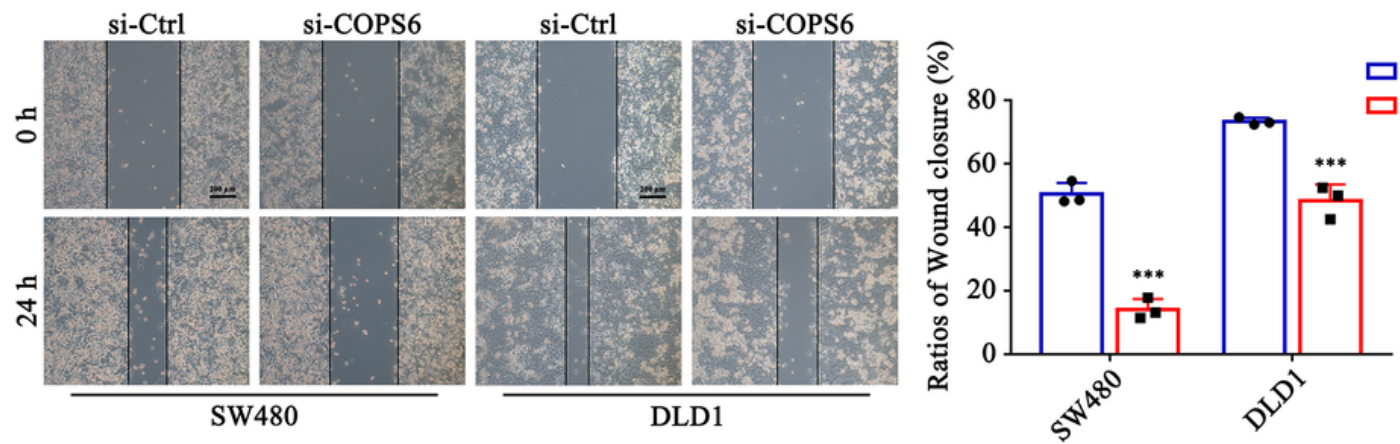

D
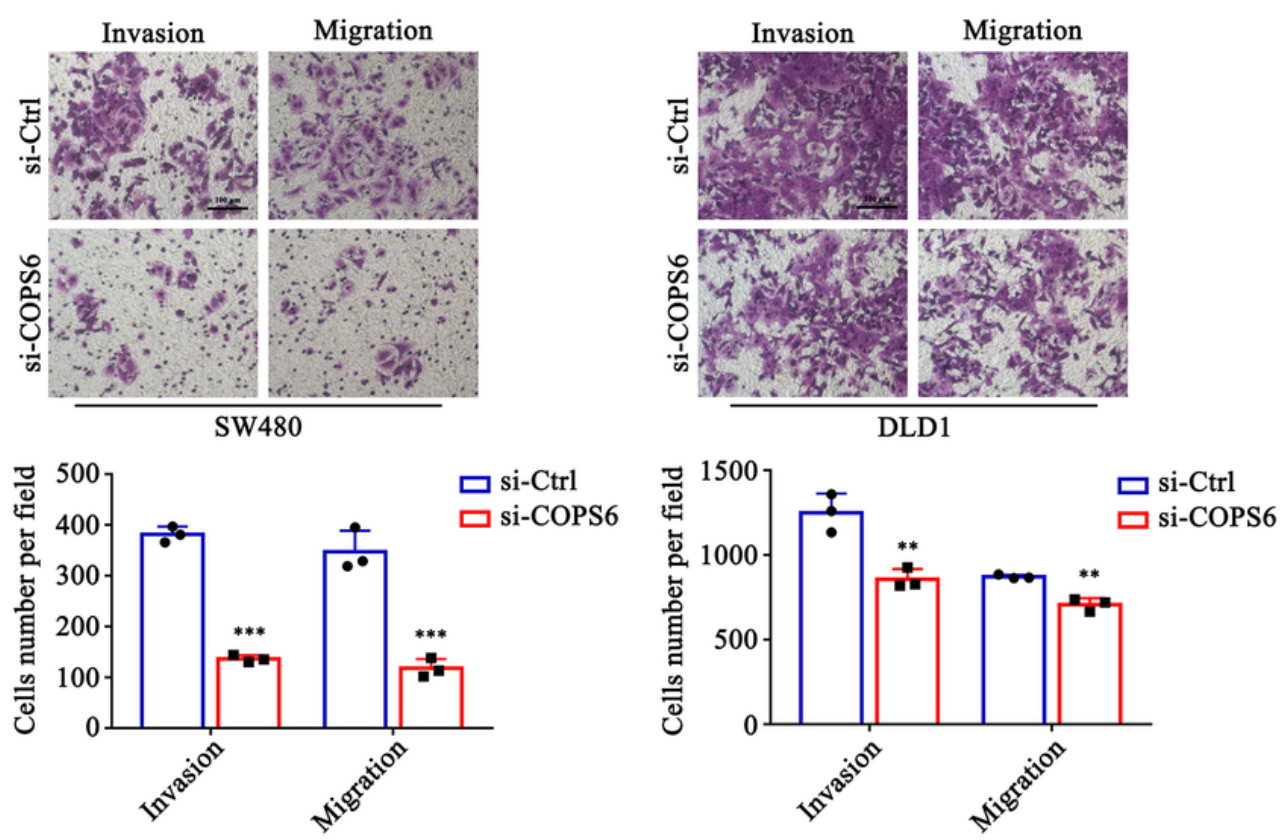

Figure 7

COPS 6 acted as a cancer promoter in CRC. (A) The basal mRNA expression of COPS 6 in CRC tissues was tested by qRT-PCR. (B) CCK-8 assays were used to evaluated the effect of COPS6 knockdown on the proliferation of SW480 and DLD1. (C) Wound healing assays were performed to assessed the migration of CRC cell with COPS6 knockdown. (D) After COPS6 being knocked down by siRNA, cell transwell assays were applied to examined the invasion and

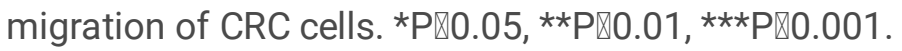



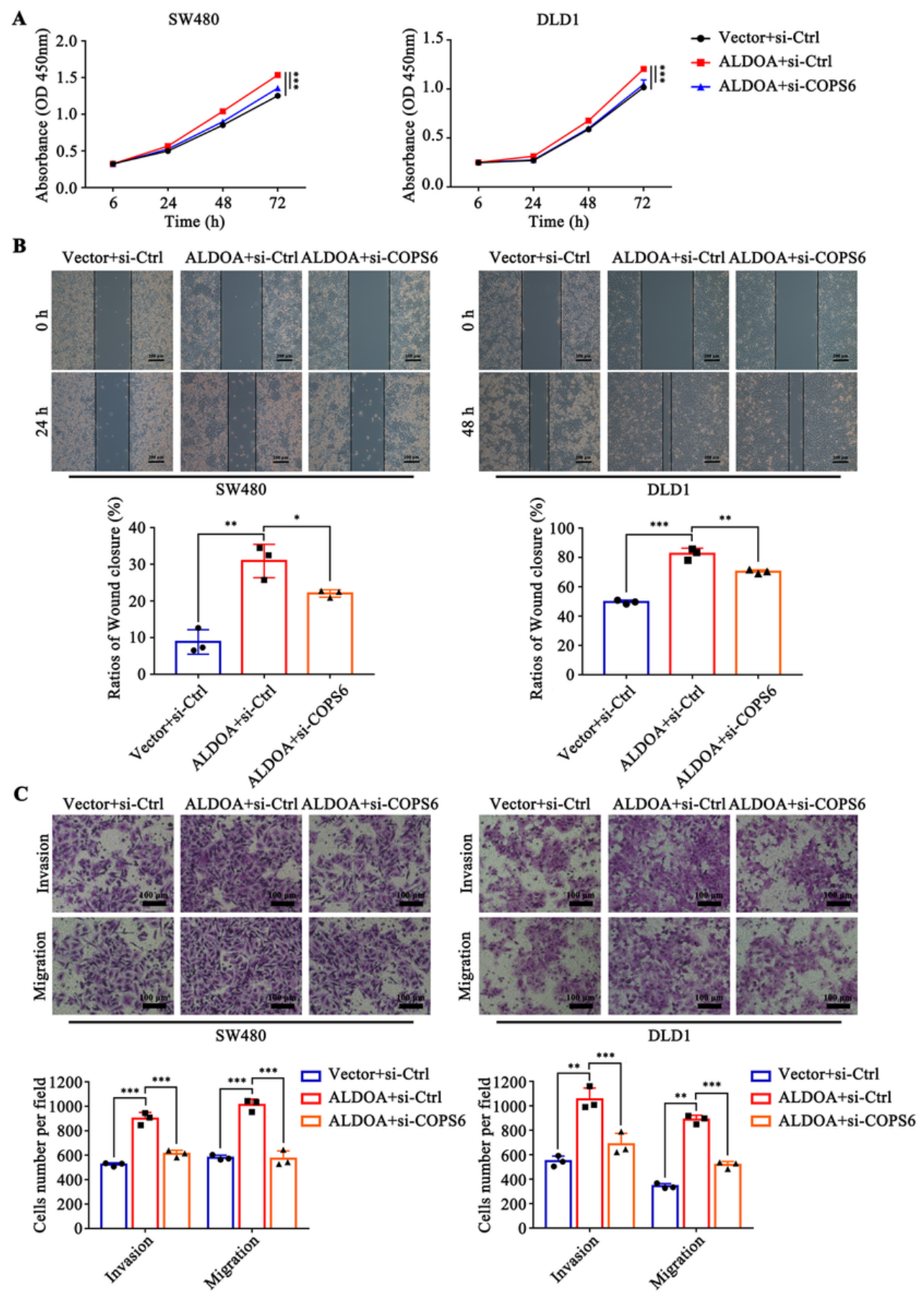

Figure 8

COPS6 knockdown reduced the promoting function of ALDOA in CRC cells. (A) CCK-8 assays showed downregulated COPS6 weaken the promotion effect of ALDOA on CRC cell proliferation. (B, C) The migration and invasion of SW480 and DLD1 were tested and quantified by wound healing and transwell assays. The stable ALDOA overexpression CRC

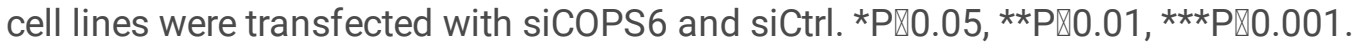


$\mathbf{A}$

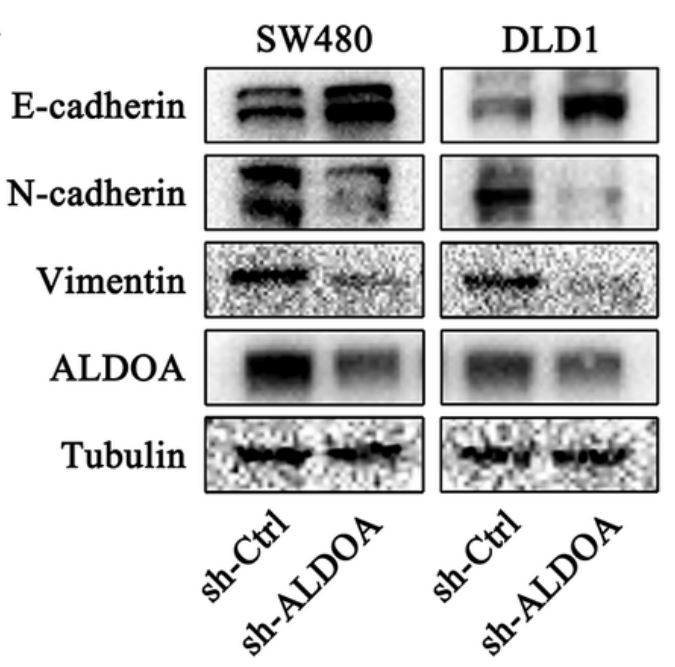

$\mathbf{C}$

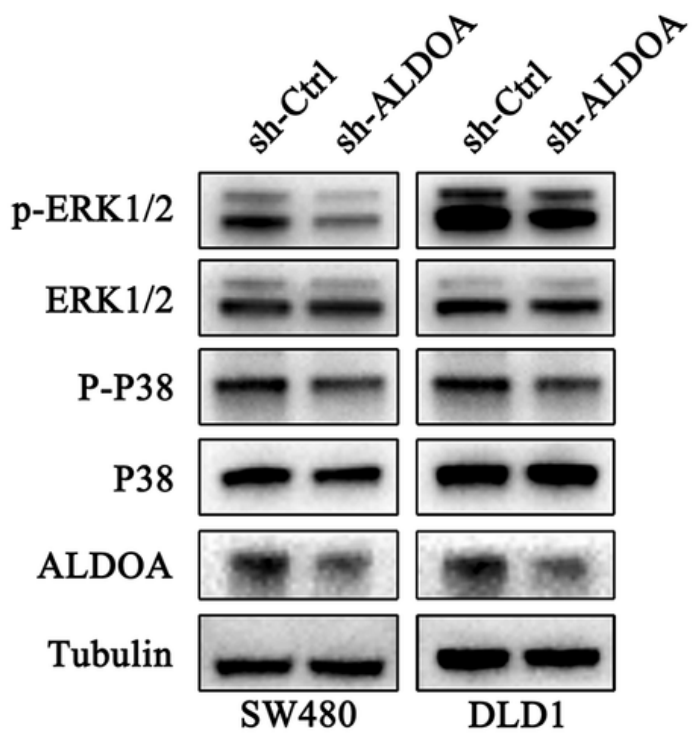

B

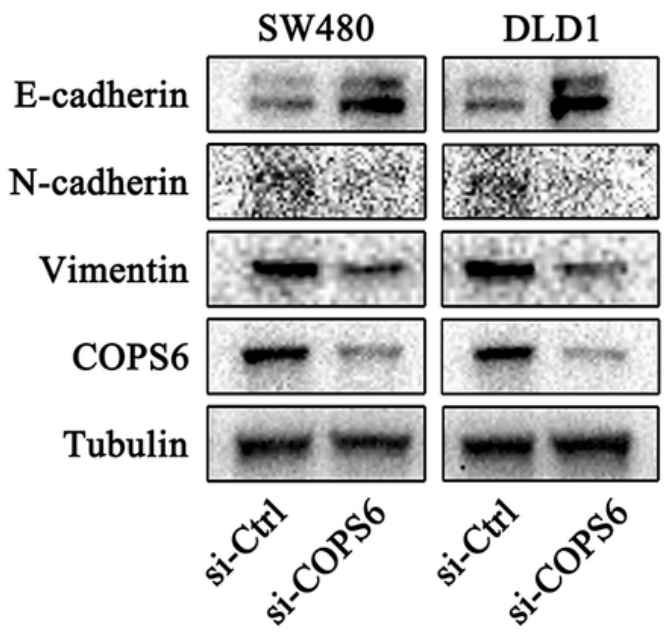

D

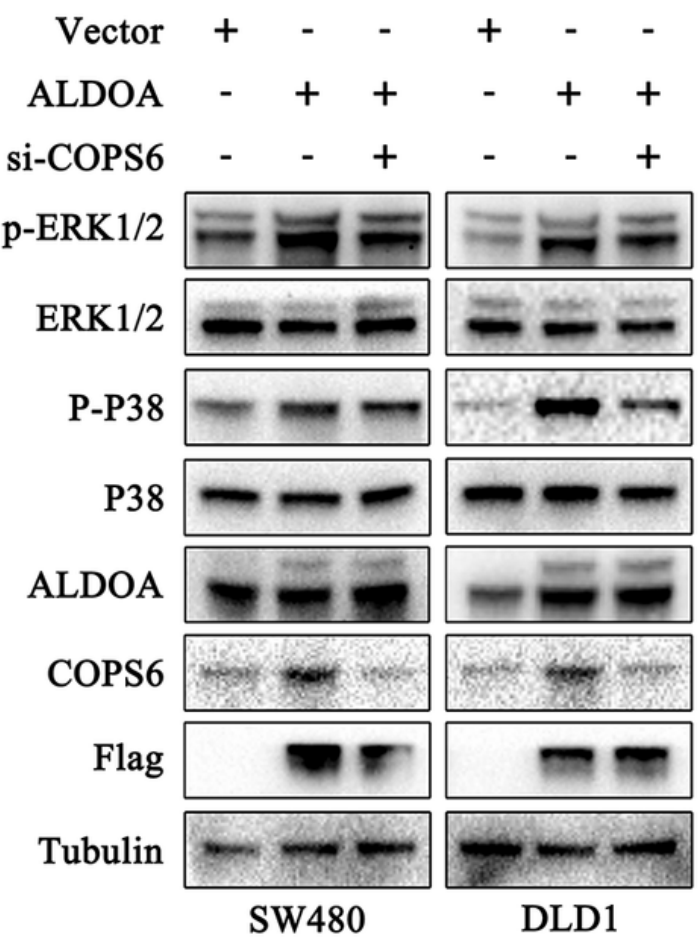

Figure 9

COPS6 was required for ALDOA accelerating CRC progression through EMT and MAPK signaling pathway. (A, B) Western blotting analysis were used to detected the expression of the indicated proteins in EMT program in CRC cells treated with sh-ALDOA and si-COPS6. (C, D) The expression of key proteins in MAPK signaling pathway were assessed by western blotting.

\section{Supplementary Files}

This is a list of supplementary files associated with this preprint. Click to download. 
- Sfigure1.tif

- Sfigure2.tif

Page 24/24 